\title{
Formación inicial de profesores de ciencias desde los enfoques CTSA y las concepciones Andinas de Vivir bien y Buen vivir
}

\author{
Jorge Enrique Ramírez Martínez ${ }^{1}$ \\ María Alejandra Beltran Penagos ${ }^{2}$
}

Recibido: 02-02-2017

Aceptado: 17-04-2017

\section{RESUMEN}

Este artículo presenta una investigación en el ámbito de la formación de profesores de ciencias llevada a cabo en un escenario no convencional de enseñanza aprendizaje, tipo curso electiva, en la Facultad de Ciencia y Tecnología de la Universidad Pedagógica Nacional denominado "Las relaciones ciencia, tecnología, sociedad y ambiente (CTSA) en la formación de profesores de ciencias". En el desarrollo de esta propuesta de formación e integración curricular, se realizaron actividades de innovación pedagógica y de apropiación social de saber y conocimiento alrededor de temáticas emergentes en la enseñanza como lo son las cuestiones sociocientíficas, las relaciones CTSA y los paradigmas andinos del Buen vivir y Vivir bien, en clave de la formación inicial de licenciados en ciencias de la naturaleza como la Física, la Química y la Biología, y de interdisciplinas como la Electrónica y el Diseño Tecnológico.

Palabras clave: Formación inicial de profesores, enfoque CTSA, transdisciplinariedad, Vivir bien y Buen vivir, Investigación como Estrategia Pedagógica.

1. Licenciado en Física y Magister en Educación, Universidad Pedagógica Nacional Bogotá, Especialista en Currículo y Pedagogía, Universidad de los Andes; Docente en la Facultad de Educación de la Universidad Santo Tomás.

Correo electrónico: kikeupn@gmail.com

ORCID: 0000-0002-1171-9710

2. Licenciada en Química, Universidad Pedagógica Nacional - Bogotá; Docente investigadora en el Centro de Investigación y Formación para la Educación Superior - CEINFES.

Correo electrónico: male.belp@gmail.com

ORCID: 0000-0003-4546-8224 


\title{
Initial training of science teachers from the CTSA approaches and the Andinean conceptions of Living well and Well living
}

\begin{abstract}
This article presents a research in the field of the training of science teachers carried out in an unconventional scenario of teaching learning, elective course type, in the Faculty of Science and Technology of the National Pedagogical University called "The relations science, technology, Society and the environment (CTSA) in the training of science professors". In the development of this proposal for curricular training and integration, pedagogical innovation activities and social appropriation of knowledge and knowledge were carried out around emerging issues in teaching such as socio-scientific issues, CTSA relations and paradigms Andinos of Good Living and Living Well, in the key of the initial formation of graduates in natural sciences such as Physics, Chemistry and Biology, and of interdisciplines such as Electronics and Technological Design.
\end{abstract}

Keywords: Initial Teacher Training, CTSA Approach, Transdisciplinarity, Living Well and Good Living, Research as a Pedagogical Strategy.

\section{Objetivo}

Presentar resultados de investigación - acción en el aula en la formación en educación superior en torno a un caso de formación inicial de profesores de ciencias. Con ello se pretende mostrar un significativo proceso de integración curricular en la Educación Superior desde la comprensión de enfoques y paradigmas que abren posibilidades en la formación de profesores de ciencias. 


\section{Marco teórico}

El interés por involucrar los enfoques CTSA en la formación de profesores de ciencias es fruto de una transformación de los contenidos científicos que se tratan en la escuela (Candela, 2006, pp. 804 - 807) y de la discusión sobre el lugar del saber científico y del conocimiento científico en el aula (Pozo \& Gómez, 2006, pp. 12 - 18). En esta mirada contemporánea sobre la formación, se permea la labor del profesor de ciencias y se presenta la necesidad de reflexionar en la enseñanza de las ciencias los aspectos relacionados con la imagen pública de la ciencia en la ciudadanía y su correspondiente impacto en la popularización del suceso científico y su respectiva socialización y divulgación en los medios de comunicación masiva (Daza \& Arboleda, 2007, pp. 113 - 114).

Del mismo modo que se procede conceptualmente, esta propuesta de formación con el enfoque CTSA, examina la incidencia de articular este discurso con la concepción de Vivir bien y Buen vivir para un caso de educación superior en la formación de profesores de ciencias. Para ello, se consideran los aportes y trabajos investigativos de la UNESCO y del Instituto Internacional para la Educación Superior en América Latina y el Caribe - IESALC sobre buen vivir y educación superior (Matto, 2009, pp. 57) y la perspectiva filosófica y práctica del paradigma vivir bien y buen vivir de la Coordinadora Andina de Organizaciones Indígenas - CAOI (Hunacuni \& Quispe, 2010, pp.7).

Epistemológicamente en esta propuesta se evidencia que hay dos paradigmas en pugna, cada uno de ellos con implicaciones en las maneras de ver, pensar y actuar en la educación en general y en la enseñanza aprendizaje de las ciencias en particular. Se trata, de dos cosmovisiones, una occidental anglosajona y eurocéntrica principalmente y otra andina, latinoamericana, milenaria y por ello, si se quiere indígena. Aparentemente son posturas irreconciliables y con dificultad de diálogo en la formación de profesores dado que en la visión occidental los problemas ambientales, la noción de desarrollo y los procesos tecnológicos no son necesariamente centrados desde el respeto a todas las formas de vida, en la que se incluye a nuestra especie como parte del ambiente.

Pedagógicamente se abordan inquietudes y cuestionamientos que relacionan la educación para una cultura científica (Sanz \& López, 2012, pp.43), la relación ciencia y ciudadanía (UNESCO; MEN; MCT, 2006, 
pp.135) y la resistencia al cambio en la alfabetización científica y docencia (Aguilar \& Ortega, 2008, pp. 180). Así como el problema de si vale la pena aprender, enseñar y problematizar la didáctica de las ciencias de la naturaleza (Tricárico, 2005, pp. 13), desde el punto de vista de las presencias y ausencias didácticas en los programas acreditados en la formación inicial de profesores de ciencias en Colombia (Gallego, Pérez, \& Torres, 2004, pp. 224 - 229).

La propuesta de este curso electivo, combina diferentes tipos de aprendizajes que son retomados en esta experiencia investigativa, principalmente el colaborativo, por indagación, el situado y el problematizador. Cada uno de estos aprendizajes busca fortalecer el análisis de casos que involucran la ciencia escolar y el desarrollo de habilidades y capacidades para resolver un determinado problema, que sea de interés para el estudiante, de manera que se facilite y adquiera un rol más activo y participativo en el aprendizaje. Sobre estos aprendizajes, según Manjarrés \& Mejía. (2012), se puede mencionar:

Aprendizaje situado: Este aprendizaje cuestiona la mirada del aprendizaje centrado en lo cognitivo, señala la necesidad de recuperar esos otros aspectos que lo hacen posible y para ello plantea que el aprendizaje sea más social que individual, y que su construcción se desarrolla con herramientas sociales más vinculadas a los sujetos, forjando pertenencia e identidad.

Aprendizaje colaborativo: En esta visión se toman las premisas de las concepciones pedagógicas de lo sociocultural y hace a cada miembro del grupo de trabajo responsable de las dinámicas de su aprendizaje, así como de los logros de cada uno de los otros participantes de su grupo. En esta perspectiva se enfatiza más en el proceso que en la tarea, ya que es en él donde se hace real la colaboración grupal, el acuerdo y la negociación.

Aprendizaje problematizador: En esta concepción se considera el conflicto como generador de dinámicas y condiciones de aprendizaje, por ello se tratan de recuperar esas tensiones de diferentes tipos: valorativas, cognitivas, sociales, culturales y colocarlas en la esfera de los contenidos y temas de la actividad educativa que se desarrolla, lo cual en la problematización genera lógicas de aprendizaje por descubrimiento, en cuanto el conflicto permite construir esos problemas, que se convierten en el medio para lograr los aprendizajes de contenidos, valores y actitudes permitiendo el desarrollo y crecimiento del niño. 
Aprendizaje por indagación: El aprendizaje por indagación implica involucrar al individuo en un problema y desde esta óptica, debe aportar soluciones. Dentro del ambiente de aprendizaje, pretende que el docente ayude a los estudiantes a externar todas esas grandes ideas a través de preguntas y de la indagación constante. Además, que ellos busquen con interés, penetrando en el fondo de las ideas, desarrollando esa capacidad de asombro ante la realidad, analizando, entendiendo y reflexionando.

En el marco de los aprendizajes y las prácticas pedagógicas desarrolladas en esta experiencia, se integran en cada fase procesos de reflexión, acción y participación desde dimensiones de construcción comunicativa y diálogos en el ciberespacio mediante la plataforma Edmodo. En este sentido, se concretan actividades que impactan la ciudadanía y convivencia en la escuela (Ramírez \& Mejia, 2015), ya que se le da un tratamiento al conocimiento científico como objeto de controversia y debate desde una construcción crítica del impacto de las nuevas tecnología en nuestra cotidianidad

\section{Abordajes metodológicos}

El punto de partida metodológico es la contrastación documental en fuentes especializadas sobre las temáticas, conceptos y enfoques presentados en el espacio académico y que conforman esta propuesta de formación. Esta revisión implicó la búsqueda en las bases de datos en línea de EBSCO, Latindex, Scielo, Redalyc, Publindex; en los metabuscadores de Google Académico; y en los catálogos y colecciones en línea de las bibliotecas de la Universidad Pedagógica Nacional; con las que se logró consolidar un buen número de artículos, libros, tesis, trabajos de grado, videos y monografías, que resultan pertinentes para dar soporte documental acorde a los fines de la propuesta curricular.

Se plantean entonces, algunas de las ventajas de articular las relaciones CTSA como evolución del enfoque CTS (Aikenhead, 2005) con el paradigma del Buen vivir y Vivir bien latinoamericano (Hunacuni \& Quispe, 2010). Evidenciando que metodológicamente este trabajo está íntimamente relacionado con la sistematización de experiencias educativas por medio de la recolección de evidencias, el análisis del currículo, los productos de las actividades y las discusiones realizadas con los profesores en formación inicial, en cada una de las sesiones y versiones de la electiva. Las prácticas de las que derivan las experiencias 
que se abordan en este artículo son entendidas por los profesores como método para la producción de conocimiento en estrecha relación con las acciones, discursos y visiones de mundo (Sánchez, 2017). En coherencia con esta noción de sistematización, esta se concibe "como práctica investigativa que recupera saberes subyacentes en las experiencias adelantadas por sujetos, en la que sus voces, sentimientos, sentidos y apuestas, proporcionan rutas y posibilidades de agencia y autogestión en el orden social, organizativo, educativo y epistemológico" (Cogollo, 2016, p. 62), aspectos que se involucran en los diseños curriculares de las diferentes versiones del espacio electivo que vivenciaron los profesores en formación.

Es preciso anotar que la contrastación entre la revisión bibliográfica, el diseño curricular inicial propuesto para la electiva, las percepciones de los profesores en formación inicial y los productos de cada semestre; se conjugaron en un proceso que retoma la investigación acción (Elliott, 1990) como modelo cíclico de mejora continua de las prácticas y de las actividades sesión a sesión en la clase, dentro y fuera del currículo, diferenciando el currículo oficial del operativo y a su vez integrando las emergencias del currículo oculto.

\section{Propuesta de formación y diseño del currículo}

Las características de la primera versión de currículo para el espacio de formación tienen que ver con la propuesta de ejercicios por fuera del aula de clase, en donde los estudiantes realizan búsquedas en bases de datos institucionales y las fuentes disponibles en las bibliotecas, revistas especializadas, trabajos de grado y tesis sobre los enfoques CTSA en la educación en general y en la formación de profesores de ciencias en particular. Como resultado de este ejercicio, en la tabla 1 se muestran las revistas colombianas especializadas en este campo que consultaron los estudiantes. Además encontraron varios trabajos de grado en torno a este enfoque, y cada estudiante escribió un resumen analítico educativo organizando el resultado de su búsqueda, el cual posteriormente fue socializado en plenaria. 
Tabla 1. Lista de revistas colombianas especializadas en el campo CTSA.

\begin{tabular}{|c|c|c|c|}
\hline Institución & Nombre de la revista & ISSN & Enlace \\
\hline $\begin{array}{c}\text { Empresa Colombiana } \\
\text { de Petróleos - } \\
\text { ECOPETROL }\end{array}$ & $\begin{array}{c}\text { CT\&F - CIENCIA, } \\
\text { TECNOLOGÍAY } \\
\text { FUTURO }\end{array}$ & $0122-5383$ & $\begin{array}{c}\text { http://www.scielo.org.co/ } \\
\text { scielo.php?script=sci_ } \\
\text { serial\&pid=0122- } \\
5383 \& \text { Ing=es }\end{array}$ \\
\hline $\begin{array}{c}\text { Instituto Tecnológico } \\
\text { Metropolitano de } \\
\text { Medellín }\end{array}$ & $\begin{array}{c}\text { TRILOGÍA. CIENCIA, } \\
\text { TECNOLOGÍAY } \\
\text { SOCIEDAD }\end{array}$ & $2145-4426$ & $\begin{array}{c}\text { http://itmojs.itm.edu.co/ } \\
\text { index.php/trilogia/ }\end{array}$ \\
\hline $\begin{array}{c}\text { Universidad Libre de } \\
\text { Colombia }\end{array}$ & $\begin{array}{c}\text { SABER, CIENCIAY } \\
\text { LIBERTAD }\end{array}$ & $1794-7154$ & $\begin{array}{c}\text { http://sabercienciaylibertad. } \\
\text { com/ojs/index.php/scyl }\end{array}$ \\
\hline $\begin{array}{c}\text { Escuela Colombiana de } \\
\text { Carrera Industriales }\end{array}$ & TECCIENCIA & $1909-3667$ & $\begin{array}{c}\text { http://tecciencia.ecci.edu.co/ } \\
\text { index.php/TECCIENCIA }\end{array}$ \\
\hline $\begin{array}{c}\text { Universidad } \\
\text { Pedagógica Nacional }\end{array}$ & $\begin{array}{c}\text { TECNE EPISTEME Y } \\
\text { DIDAXIS - TED }\end{array}$ & $0121-3814$ & $\begin{array}{c}\text { http://revistas.pedagogica. } \\
\text { edu.co/index.php/TED }\end{array}$ \\
\hline $\begin{array}{c}\text { UNIPAZ - Instituto } \\
\text { Universitario de la Paz }\end{array}$ & REVISTA CITECSA & $2027-6745$ & $\begin{array}{c}\text { http://www.unipaz.edu.co/ } \\
\text { ojs/index.php/revcitecsa }\end{array}$ \\
\hline
\end{tabular}

Se incluye en los resultados de dicha búsqueda el portal web de la Organización de Estados Iberoamericanos para la Educación, la Ciencia y la Cultura (OEI), en el cual se divulgan trabajos de toda Latinoamérica y el Caribe sobre los temas de interés en el curso electivo y varios de los documentales y vídeos cortos allí disponibles que hicieron parte de los materiales utilizados en las discusiones del curso.

Dentro de la metodología de clase también se encuentra la participación en diferentes eventos académicos de socialización y apropiación del conocimiento, dentro de los cuales estuvo la conferencia "Huella de carbono: disminuirla, un reto para la sostenibilidad de las organizaciones"; las conferencias institucionales organizadas por el Departamento de Química denominadas "Viernes de la didáctica de las ciencias", el IV Seminario Internacional de Investigación sobre la Calidad de la Educación, el conversatorio sobre "Educación y Cerebro" con el Dr. Rodolfo Llinás; y otros eventos sugeridos como la "II Jornada de la Memoria Pedagógica; sobre el movimiento del movimiento pedagógico" y la conferencia "Redes complejas: aplicaciones de la física a sistemas sociales, biológicos y artificiales".

Por otra parte, se propone que los recursos audiovisuales sean un insumo para propiciar reflexiones alrededor de diversos casos relacionados con la ciencia, la tecnología, la sociedad y el ambiente. El primer recurso que se visualizó fue el documental Surplus (Gandini, 2003) acerca de la 
sociedad del consumo; seguido por el Documental 9.70 (Solano, 2013) sobre la propiedad intelectual de las semillas en Colombia; la película Home (Arthus-Bertrand, 2009) que presenta una visión global del problema ambiental causado por el consumo, la película También la lluvia (Bollaín, 2010) que muestra las luchas sociales por el agua y el documental Una verdad incómoda (Guggenheim, 2006) sobre los efectos del calentamiento global.

En clave de los planteamientos epistémicos que tratan a la investigación acción como posibilidad de aporte a la construcción de un currículo negociado (Pizarro \& Milagros, 2013), se plantea la segunda y tercera versión del diseño curricular desde el estudio de casos como técnica didáctica o también denominado aprendizaje basado en casos (ITESM, 2006). En esta versión se realiza la apertura de la electiva con una conferencia titulada "Escenarios de apropiación social de la ciencia y la tecnología en la niñez y juventud. Experiencias de maestros desde el Programa Ondas de Colciencias", con la que se busca incentivar a la construcción de preguntas de investigación como punto de partida. Se socializó en esta conferencia cómo los niños y jóvenes apropian la cultura, la ciencia y la técnica a partir de procesos de investigación, mostrando casos concretos de proyectos que derivaron en productos de emprendimiento e innovación educativa, social o ambiental.

Posterior a ello, los profesores en formación inicial realizaron la lectura del texto base sobre Ambiente y Buen Vivir latinoamericanos (Hunacuni \& Quispe, 2010), la cual resultó muy interesante y derivó en discusiones sobre las visiones de CTSA presentes en este paradigma epistemológico. Con estos insumos de lecturas, búsquedas y análisis de textos se invita a los estudiantes a participar en una salida de campo a los departamentos de Cundinamarca, Tolima, Antioquia y Caldas, para la cual se diseña una hoja de ruta a desarrollar, que tiene varios niveles de complejidad de menores a superiores en cuanto a las capacidades y habilidades para observar, describir, analizar, interpretar y proponer. Dentro de los objetivos de la salida de campo se encuentra que a partir de ella los profesores en formación identifiquen y analicen propuestas curriculares con orientación CTSA dirigidas a la enseñanza de las ciencias, y además que reconozcan y describan las implicaciones sociales, económicas, políticas, culturales y educativas asociadas a las problemáticas identificadas en cada lugar, para que se desarrollen habilidades de pensamiento y acción de tipo crítico, reflexivo y propositivo en relación con la enseñanza de las ciencias. 
Junto a la hoja de ruta, previo a la salida de campo, se comparten con los estudiantes lecturas que abordan algunas de las problemáticas que se conocerán al vivenciar los diferentes espacios del recorrido. Estas lecturas son un insumo importante para enriquecer las franjas de plenaria y las actividades propuestas al final de cada día. En la primera plenaria se indaga sobre ¿Qué aspectos entre las relaciones ser-naturaleza destaca desde una perspectiva CTSA?, ¿Cuáles fueron las problemáticas y los impactos socio-ambientales percibidos en cada estación? y ¿Qué relaciones identifica entre las distintas estaciones y las lecturas propuestas? Ante estos interrogantes los estudiantes mostraron sus habilidades para identificar y describir varias de las problemáticas presenciadas y para reconocer la responsabilidad de las actividades humanas en el impacto ambiental sobre las fuentes hídricas, la flora y la fauna.

En una actividad posterior se invita a plantear una pregunta de investigación por grupos y diseñar una ruta metodológica para desarrollar su pregunta. Para ello debieron escoger uno de los siguientes ejes: currículo, sociedad, enculturación científica, representaciones sociales, apropiación social del conocimiento o ambiente. Este ejercicio arrojó frutos inesperados, que derivaron en discusiones principalmente sobre el para qué de la CTSA en un desarrollo social y su relación con el respeto de todas las formas de vida, asunto crucial del paradigma del Buen Viviry Vivir Bien. En el marco de estas deliberaciones, diálogos y actividades de clase surge la invitación a que cada uno realice una propuesta de diseño e implementación de una sesión de clase dirigida por ellos en la que se integren las dos perspectivas.

\section{Ámbitos de la sistematización}

Con la sistematización de la información obtenida en los ejercicios iniciales de búsqueda de fuentes, se realiza una discusión sobre el lugar de la divulgación de la ciencia y de la conformación de comunidades especializadas de producción de sabery conocimiento, que lleva a concluir que aún la ciencia se produce y se divulga en el país en centros (distritos y capitales) más que en periferias (municipios). Otra importante conclusión de esta discusión es que las publicaciones revisadas, a excepción de la revista TED, no incluyen una línea editorial desde lo educativo.

Como resultado de las reflexiones de los estudiantes a partir de la asistencia a los eventos científicos y el análisis de los recursos 
audiovisuales, se dilucida la comprensión de la ciencia como una única fuente para la construcción de conocimiento, evocando equivocaciones y destrucción por parte del hombre, para la explotación de recursos; la ciencia como actividad humana responsable de la contaminación y la escasez. La tecnología es vista como los instrumentos, las herramientas - las máquinas para producir bienes y servicios que satisfagan las necesidades particulares y en cuanto a la concepción de ambiente, éste es entendido separadamente como un ambiente social, en el que convergen relaciones de poder entre los seres humanos, y un ambiente naturaleza, que viene a significar una fuente de recursos y materia prima. Por consiguiente, en cuanto a la formación de profesores de ciencias, los estudiantes consideran necesario replantear las concepciones establecidas acerca de la interacción del hombre con el medio ambiente y la utilización de la ciencia y la tecnología como elemento mediador en esta relación por las implicaciones que esto tendría en el desempeño en las prácticas pedagógica (Sánchez, 2015). De modo que se suscitan los siguientes interrogantes: ¿Podemos educar en total coherencia para la convivencia del hombre y la naturaleza? ¿Cuál es el compromiso individual y colectivo que estamos afrontando actualmente? ¿Hemos comprendido que el problema ambiental nos afecta a todos ya que somos "una gran familia"?

Estas preguntas y los comentarios frente a la lectura del texto de Hunacuni \& Quispe (2010) mostraron que el paradigma andino plantea una idea de respeto por la naturaleza y las demás personas, en la que no existe una necesidad de acumulación de bienes sino una economía que se basa en las necesidades y aportes de los miembros de la comunidad como alternativa al individualismo, concibiendo el desarrollo como una forma de reconocimiento de la diversidad cultural que no atente contra el ambiente.

En socialización de las actividades en la salida de campo, se evidenció que los estudiantes tienen dificultad en plantearuna pregunta de investigación y en la identificación de un problema, la ubicación en un contexto/ población/comunidad, la delimitación de la pregunta de acuerdo a sus posibles alcances y perspectivas, y la construcción de una metodología o ruta que correspondiera con el objetivo general. En la plenaria final, se consideraron los aprendizajes generales de la salida dentro de los que se destaca que el aprendizaje en espacios no convencionales constituye un aporte muy importante a la formación de profesores en tanto que da la oportunidad de vivenciar los impactos inmersos en el marco de las 
relaciones CTSA para fortalecer el pensamiento crítico y fortalecer las habilidades investigativas.

\section{Conclusiones y perspectivas de análisis}

Uno de los conceptos cruciales presentes en cada una de las sesiones de la electiva y de características polisémicas es el de ambiente. Los profesores en formación inicial afirmaban antes del desarrollo de las actividades que el ambiente está en íntima y estrecha relación con su propia concepción de ciencia, tecnología y sociedad. A pesar de ello, lo consideran por fuera de su propia existencia y de su realidad educativa.

Aunque este artículo no se centra en describir la transformación de ideas que tuvieron los participantes, si resalta el hecho que en su gran mayoría se percibe una ruptura epistémica, por el encuentro de ellos con las nociones de ambiente andinas, latinoamericanas, presentes en el Buen Vivir Ecuatoriano y Boliviano. En este sentido, traer a colación el caso de la ruptura epistemológica, modificabilidad o cambio conceptual sobre la concepción de ambiente, nos pone de presente los retos de una formación de profesores de ciencias abordada desde referentes transdisciplinares, ya que, la polisemia presente en este caso, deja ver la necesidad de transformar y superar los debates de las interdisciplinas, multidisciplinas y transdisciplinas en la enseñanza de las ciencias, mostrando la urgente necesidad de construir discursos en el aula que reinterpreten los saberes y conocimientos científicos en vía de construir un pensamiento lo más auténtico, situado y contextual en el aula.

Lo anterior, implica articular las ciencias con las humanidades y las artes. Un antecedente de ello es la propuesta de Prigogine (1990), premio Nobel de Química en 1977, denominada La nueva alianza. En la que se propone desde el seno de la ciencia positivista, un encuentro de saberes para una ciencia del devenir, en la cual se superen las contradicciones de las dos culturas, al incorporar a la vida y la historia, en la concepción de ciencia de la comunidad científica.

Muchas implicaciones se pueden asociar a esta propuesta de Prigogine para la formación y la visión de la ciencia en el aula. En este espacio académico fue de gran aporte para la validación de los saberes populares y de los conocimientos ancestrales, ya que aunque parezca extraño hubo necesidad de discutir sobre la validación de los mismos y su lugar 
en el saber escolar de la modernidad. Como lo evidencia también el trabajo de Alfaro, Fernández, \& González (2015), es posible realizar análisis de las realidades a partir de procesos participativos y diálogos transdisciplinarios, que permitan la complementariedad entre las ciencias que se ocupan de los aspectos tangibles con las voces y conocimientos de los protagonistas de los procesos; en donde la Universidad con el debate educativo sea un puente para generar aprendizajes.

En cuanto a la pregunta sobre la posibilidad de proponer una didáctica de las ciencias desde las relaciones CTSA, se pudo evidenciar con esta práctica formativa que "frente a las relaciones CTS y CTSA si se quiere, los profesores de ciencias de la naturaleza, junto con los demás colegas de otras comunidades de especialistas, estaríamos avocados a un problema didáctico con campo fértil en la alfabetización científica y cultural, de hecho la sola alusión al cambio climático como un problema complejo que debe abordarse de manera urgente y necesaria desde el aula (Gallego, Rocha, \& Castro, 2009), ratifica la configuración de un problema que abriga las dimensiones: científica, tecnológica, social, ambiental y cultural" (Franco, González, \& Ramírez, 2010), por ello esta investigación en una aplicación que desde estos enfoques problematiza la formación inicial de profesores de ciencias y presenta caminos para su inclusión en un currículo integrado.

\section{Referencias bibliográficas}

Aguilar, M., \& Ortega, J. (2008). Alfabetización científica, epistemología y docencia. Enseñanza e Investigación en Psicología, 177-185.

Aikenhead, G. (2005). Educación Ciencia-Tecnología-Sociedad (CTS): una buena idea como quiera que se le llame. Educación Química, 2(16), 114-124.

Alfaro, J., Fernández, C., \& González, M. (2015). La transdisciplinariedad una herramienta para apuntar al Buen Vivir. Polis, 40, 1-15. doi:10.4000/polis.10896

Arthus-Bertrand, Y. (Dirección). (2009). Home [Película].

Bollaín, I. (Dirección). (2010). También la lluvia [Película].

Candela, A. (28 de julio-septiembre de 2006). Del conocimiento extraescolar al conocimiento científico escolar: Un estudio etnográfico en aulas de la. (C. M. Educativa, Ed.) Revista Mexicana de Investigación Educativa, 11(30), 797-820. 
Cogollo, C. (2016). Trayectorias de la sistematización de experiencias. Su constitución como posibilidad de pensar la producción de conocimiento en escenarios académicos. Revista Interamericana de Investigación, Educación y Pedagogía-RIIEP, 9(1), 54-66.

Daza, S., \& Arboleda, T. (2007). Comunicación pública de la ciencia y la tecnología en Colombia: ¿políticas para la democratización del conocimiento? Signo y Pensamiento, 36(50), 99-125.

Elliott, J. (1990). La investigación-acción en educación. Madrid: Ediciones Morata.

Franco, R., González, C., \& Ramírez, J. (2010). Las relaciones CTSA: un campo de investigación. Revista Teckne, 6-8.

Gallego, A., Rocha, P., \& Castro, E. (2009). El cambio climático un problema complejo. Tecné, Episteme y Didaxis, 552-557.

Gallego, R., Pérez, M., \& Torres, L. (2004). Formación inicial de profesores de ciencias en Colombia: un estudio a partir de programas acreditados. Ciência \& Educação, 219234.

Gandini, E. (Dirección). (2003). Surplus [Película].

Guggenheim, D. (Dirección). (2006). Una verdad incómoda [Película].

Hunacuni, F., \& Quispe, M. (2010). Buen Vivir / Vivir Bien. Filosofía, políticas, estrategias y experiencias regionales andinas. Lima.

ITESM. (1 de junio de 2006). Las estrategias y técnicas en el rediseño. Recuperado el 10 de abril de 2013, de http://sitios.itesm.mx/va/dide2/documentos/casos.PDF

Matto, D. (2009). Educación Superior, Colaboración Intercultural y Desarrollo Sostenible/Buen Vivir. Experiencias en América Latina. Caracas: UNESCO-IESALC.

Manjarrés, \& M. R. Mejía. (2012). Niños, niñas y jóvenes investigan. Lineamientos pedagógicos del Programa Ondas (págs. 88-122). Edeco Ltda.

Manjarrés, M., \& Mejía, M. R. (2013). Xua, Teo y sus amigos en la onda de la investigación. Guía de la investigación y de la innovación del Programa Ondas. Bogotá D.C: Chigüiro editores S.

Mejía, M. R., \& Manjarrés, M. E. (2013). La investigación como estrategia pedagógica, una propuesta desde el sur. Bogotá: Desde Abajo.

Pizarro, G., \& Milagros, V. (3 de Junio de 2013). La investigación - acción como aporte a la construcción de un currículo negociado. Recuperado el 16 de Junio de 2014, de: http://tesis.pucp.edu.pe/repositorio/handle/123456789/4741

Pozo, J., \& Gómez, M. (2006). Aprender y Enseñar Ciencia. Madrid: Ediciones Morata, S.L. 
Prigogine, I. (1990). La nueva alianza: metamorfosis de la ciencia. Madrid: Alianza universidad.

Ramírez, J., Mejía, M., Cantor, H. \& González, L. (2015). Educación para la Ciudadanía y la Convivencia-Ciclo Cinco: Ciberciudadanía para la paz. Una aldea digital de la que haces parte.

Sánchez, A. (2015). Concepciones de los docentes en formación y sus implicaciones en el desempeño en las prácticas pedagógicas. Revista Interamericana de Investigación, Educación y Pedagogía-RIIEP, 8(2), 263-277.

Sánchez, C. (2017). Una propuesta epistemológica para la sistematización de experiencias generada desde la reflexión sobre la práctica. Revista Interamericana de Investigación, Educación y Pedagogía, RIIEP, 9(2). 11-26. Recuperado de http://revistas. usta.edu.co/index.php/riiep/article/view/3612/3519

Sanz, N., \& López, J. (2012). Cultura científica para la educación del siglo XXI. Revista iberoamericana de educación, 35-59.

Solano, V. (Dirección). (2013). Documental 9.70 [Película].

Tricárico, H. (2005). Didáctica de las ciencias naturales. ¿Cómo aprender? ¿Cómo enseñar? Buenos aires: Bonum.

UNESCO; MEN; MCT. (2006). Ciencia e cidadania: Seminário Internacional Ciencia de Qualidade para Todos. Brasilia: UNESCO. 